\title{
Aproximaciones al estudio del comportamiento de los productores agropecuarios en el Chaco Seco
}

\author{
Matías E. Mastrangelo ${ }^{1,2}$ \\ ${ }^{1}$ Grupo de Estudio de Agroecosistemas y Paisajes Rurales (GEAP), Unidad Integrada Balcarce (EEA INTA Balcarce - Facultad \\ de Ciencias Agrarias, Universidad Nacional de Mar del Plata. ${ }^{2}$ Consejo Nacional de Investigación Científica y Técnica \\ (CONICET).
}

\begin{abstract}
Resumen. En forma creciente, los ecólogos somos llamados a desempeñar un rol más activo en la gestión de los recursos naturales y en la planificación de los ecosistemas y paisajes rurales de nuestro país. La efectividad de la gestión y planificación depende mayormente de la capacidad para transformar los comportamientos humanos que más influyen sobre el estado de los ecosistemas y los paisajes. Por lo tanto, la capacidad de los procesos y resultados de la investigación en ecología para transformar la realidad de los territorios se puede potenciar al integrarlos con elementos conceptuales y metodológicos de las ciencias del comportamiento humano. En el territorio, los cambios en el uso y cobertura de la tierra son el resultado de una multiplicidad de decisiones que toman quienes afectan de manera directa a los ecosistemas y a aquellos que se benefician de ellos, en su mayoría productores agropecuarios. El presente trabajo revisa teorías, conceptos, métodos y aplicaciones de la psicología social para visibilizar y poner en debate sus aportes reales y potenciales a la investigación y la gestión de ecosistemas y paisajes rurales sujetos a transformación por la agricultura, los que son ilustrados con estudios en el Chaco Seco argentino. La teoría del comportamiento planificado demostró ser un modelo conceptual y analítico de gran valor sinóptico para comprender los factores motivacionales endógenos que influyen sobre la formación de las intenciones de conservar por parte de productores agropecuarios. La valoración social de servicios ecosistémicos permitió observar la diversidad de identidades y de perfiles de valoración que coexisten en el territorio. Finalmente, la superposición de curvas de compromiso e indiferencia mostró ser una herramienta simple y poderosa para vincular los compromisos ecológicos con los conflictos de preferencias, y para identificar barreras y oportunidades que le permitan a los productores agropecuarios realizar una transición hacia sistemas eficientes y aceptables de uso de la tierra.
\end{abstract}

[Palabras clave: psicología social, teoría del comportamiento planificado, servicios ecosistémicos, valoración social, compromisos, conflictos, productores agropecuarios, multifuncionalidad, bosques nativos]

\begin{abstract}
Aвstract. Contributions of human behavior study to the research and management of rural ecosystems and landscapes. In our country, ecologists are increasingly called to play a more active role in natural resource management and in rural ecosystem and landscape planning. The effectiveness of management and planning depends to a large extent on the ability to transform the human behaviors that most influence the state of ecosystems and landscapes. Therefore, the capacity of research processes and results in ecology to transform the reality of territories can be enhanced by its integration with conceptual and methodological elements of the human behavioral sciences. In the territory, changes in land use and land cover are the result of a multiplicity of decisions made by those individuals that affect and benefit from ecosystems, mostly farmers. This paper reviews theories, concepts, methods and applications of social psychology to make visible and discuss their real and potential contributions to the research and management of rural ecosystems and landscapes subject to transformation by agriculture, which are illustrated by studies in the Argentine Dry Chaco. The Theory of Planned Behavior proved to be a conceptual and analytical model of great synoptic value to understand the endogenous motivational factors that influence the formation of intentions to conserve remnant forests by agricultural producers. The social valuation of ecosystem services made it possible to observe the diversity of identities and valuation profiles that coexist in the territory. Finally, overlapping trade-off and indifference curves proved to be a simple and powerful tool for linking ecological and preference trade-offs, and for identifying barriers and opportunities for farmers to transition to land use systems that are both biophysically efficient and socially acceptable.
\end{abstract}

[Keywords: social psychology, theory of planned behavior, ecosystem services, social valuation, trade-offs, conflicts, farmers, Chaco, multifunctionality, native forests] 


\section{INTRODUCCIÓN}

En forma creciente, los ecólogos somos llamados a desempeñar un rol más activo en la gestión de los ecosistemas y paisajes rurales de nuestro país (Gurvich et al. 2009; Paruelo 2016). La efectividad de dicha gestión depende, en gran medida, de la capacidad para transformar los comportamientos humanos que impulsan con mayor fuerza los cambios en ecosistemas y paisajes. Dado que el comportamiento humano no suele formar parte de los estudios ecológicos en nuestro país, incorporar enfoques y colaborar con profesionales de las ciencias sociales resulta clave para comprender las fuerzas que impulsan el cambio ecosistémico y para aumentar nuestra capacidad de producir conocimiento útil para la gestión. El comportamiento de los productores agropecuarios es el factor que más influye sobre la estructura y el funcionamiento de ecosistemas y paisajes rurales sobre extensas regiones de nuestro país. Este comportamiento de los productores suele afectar el funcionamiento ecosistémico a diferentes escalas espaciales y temporales, ya que, entre otras cosas, modifica el tipo y la extensión de la cobertura vegetal, y con ello, la dinámica de diversos procesos ecológicos (Volante et al. 2012). Estas modificaciones son impulsadas por diversos tipos de productores agropecuarios mayormente para aumentar la producción de bienes materiales (e.g., alimentos, fibras o combustibles). De esta manera, los productores agropecuarios juegan un doble rol en los paisajes rurales al ser a la vez afectadores y beneficiarios del funcionamiento ecosistémico (Scheffer et al. 2000). Cuando las alteraciones del ecosistema producen pérdidas de biodiversidad y funciones ecosistémicas, los productores agropecuarios perjudican la producción de bienes y servicios de aquellos ecosistemas de los cuales se benefician otros actores sociales o la sociedad en su totalidad. Por ejemplo, una empresa agropecuaria que desmonta un fragmento de bosque nativo amplía la superficie cultivable y el volumen de producción de granos y carne, $\mathrm{y}$, al mismo tiempo, reduce la capacidad del ecosistema para regular el clima, controlar la erosión, proveer hábitat a organismos beneficiosos (e.g., polinizadores, controladores biológicos, etc.), entre otras funciones y servicios. Entre sus objetivos, la gestión de los ecosistemas y paisajes rurales incluye incentivar decisiones de uso de la tierra que produzcan beneficios diversos (tangibles e intangibles, públicos y privados) con el menor perjuicio posible sobre la biodiversidad y funciones ecosistémicas, de manera tal de conservar la multifuncionalidad en el largo plazo (Mastrangelo et al. 2014). En este contexto, mejorar nuestro conocimiento sobre el comportamiento de los distintos tipos de productores agropecuarios es fundamental para diseñar incentivos que sean adoptados en forma efectiva y duradera.

A escala global, la agricultura es la causa principal de la transformación de ecosistemas y paisajes rurales, y de su capacidad para proveer beneficios a la sociedad de manera sustentable (Ellis et al. 2010). En el Gran Chaco sudamericano, la conversión de ecosistemas naturales y semi-naturales para usos agrícolas y ganaderos se produjo con mayor magnitud y velocidad que en cualquier otra región del mundo durante los últimos 15 años (Hansen et al. 2013). En el Chaco Seco argentino, esta expansión de la agricultura generó la transformación de 15.8 millones de hectáreas de bosques nativos hasta 2012 (Vallejos et al. 2015). A escala regional, la deforestación resulta de la acción sinérgica de factores económicos, tecnológicos y políticos, que, facilitados por factores climáticos, penetran desde fuera de la región con el objetivo de incorporarla a un modelo de desarrollo basado en la exportación de "commodities" agrícolas. Esto genera territorios donde fronteras de deforestación impulsadas por fuerzas globales se expanden desplazando modos de vida indígenas y campesinos (Brannstrom 2009). Allí, los cambios en el uso y cobertura de la tierra son cada vez más el resultado de una multiplicidad de decisiones tomadas en forma relativamente individual e independiente por productores agropecuarios que se relacionan de formas muy distintas con la tierra que usan. En definitiva, la fragmentación del paisaje en las fronteras agropecuarias del Chaco Seco refleja, en gran medida, la fuerte fragmentación social y cultural del territorio, que resulta de una historia dominada por el conflicto en torno a la tenencia y uso de la tierra entre diferentes actores territoriales.

La producción agropecuaria en el Chaco Seco se inició con la llegada de los criollos y los colonos al final del siglo XIX y a comienzos del XX, quienes desarrollaron sistemas de baja intensidad de uso de la tierra como la ganadería "a monte" o el cultivo en pequeños predios bajo formas precarias de tenencia de la tierra (Morello et al. 2005). En los años '70 y '80 comienzan a expandirse formas de hacer agricultura con tecnologías de la llamada Revolución Verde (i.e., mejoramiento 
genético, agroquímicos, maquinaria, riego) en las zonas con mayor aptitud agrícola e infraestructura, lo cual inicia un proceso de reemplazo de productores con baja intensidad de uso de la tierra sobre pequeños predios y tierras comunales por productores con alta intensidad de uso de la tierra sobre grandes predios privados (Grau et al. 2008). Este proceso se extiende y profundiza en las últimas dos décadas con el arribo masivo de productores de la Región Pampeana, quienes acaparan tierras en el Chaco Seco e impulsan una aceleración de la deforestación y la conflictividad social como resultado de su irrefrenable ambición por ampliar la superficie cultivable con soja o pasturas para ganadería intensiva (Cáceres 2015).

\section{Abordajes para el estudio del comportamiento de los productores agropecuarios}

A escala global, y en especial en nuestro país, el estudio del comportamiento de los productores agropecuarios no fue desarrollado en forma proporcional a su importancia como impulsor del cambio ambiental. Los estudios sobre la gestión de los ecosistemas se basaron en el supuesto del Homo economicus, es decir, del actor territorial como un agente cuyas decisiones son puramente racionales y sólo están orientadas a maximizar el beneficio económico propio. A pesar de la enorme cantidad de estudios que evidencian lo inapropiado de este modelo de comportamiento humano, hoy existen herramientas de investigación y gestión de los ecosistemas que aún se basan en suponer aspectos del Homo economicus (e.g., los esquemas de pagos por servicios ecosistémicos [Levine et al. 2015]). En paralelo al auge de mecanismos de gestión ecosistémica basadas en incentivos económicos, los mecanismos de regulación del uso de los ecosistemas por parte del Estado, como el ordenamiento territorial rural, suelen basarse sobre un conocimiento somero del comportamiento de los actores territoriales (Mastrangelo et al. 2015). Frente a esta situación, resulta de gran valor tanto básico como aplicado la ampliación de las capacidades de abordaje conceptual y metodológico para estudiar el comportamiento de los productores agropecuarios. Distintas disciplinas ofrecen teorías, conceptos y métodos para tal fin. El análisis institucional permite identificar cómo el comportamiento de un grupo o un individuo es influenciado por normas y reglas establecidas por instituciones formales o informales, lo que lo hace en particular apropiado para contextos en los que la acción colectiva y las instituciones son fuertes y tienden a regir el comportamiento (Agrawal and Gibson 1999). Los modelos económicos fueron muy utilizados para comprender el manejo de los ecosistemas, aunque su énfasis en el agente puramente racional, utilitario y egoísta ha generadoexplicaciones tan elegantes como erróneas como la famosa Tragedia de los Comunes (Hardin 1968). Los modelos institucionales y económicos pueden ser útiles en análisis a grandes escalas, en los cuales los incentivos económicos y las regulaciones institucionales determinan mayormente la asignación de tierras a los distintos usos en función de aptitudes, costos y beneficios (Edwards-Jones 2006). Sin embargo, estos modelos van perdiendo validez a medida que las decisiones de uso de la tierra se analizan a escalas más finas, en las que la diversidad de comportamientos crece y los factores noeconómicos ganan cada vez más influencia (Poppenborg and Koellner 2013).

Las ciencias sociales ofrecen múltiples formas para abordar la dinámica del comportamiento de los diferentes actores territoriales. Estos abordajes varían en función de su marco teórico, metodológico y sus objetivos. En la década del sesenta emerge la ecología cultural como forma de comprender los cambios en el paisaje rural a partir del estudio del comportamiento de los actores territoriales; integra la teoría de sistemas, los métodos cualitativos de la antropología y los estudios sobre lógicas campesinas de Chajanov (1966) y Boserup (1965) (Roy Chowdhury and Turner 2006). Este énfasis en la agencia de los actores territoriales surgió en forma paralela en los estudios sobre riesgo ambiental, aunque utilizando métodos cuantitativos como en el caso de los modelos basados en agentes (Brookfield 1972). En los '80, estos abordajes fueron criticados por considerar de manera insuficiente el rol de las estructuras sociales como determinantes del comportamiento de los actores territoriales; bajo este argumento, la ecología cultural dio lugar a la ecología política y los estudios sobre vulnerabilidad, ambas con un enfoque estructuralista como la sociología rural (Blaikie and Brookfield 1987). Más recientemente, diversos enfoques disciplinares e interdisciplinares abordan en forma integrada el rol de la agencia y la estructura en los paisajes rurales, combinando métodos cualitativos y cuantitativos. Uno de ellos comparte el propósito de la psicología 
ambiental de comprender la influencia retroactiva entre el cambio ambiental y el comportamiento humano, mediada por las creencias, valores, actitudes, percepciones y lógicas de tomas de decisiones de los individuos y los grupos sociales (Meyfroidt 2013). Este enfoque adopta dichos elementos conceptuales de la psicología social con el objetivo de comprender y predecir el comportamiento de los actores territoriales bajo la influencia de factores tanto endógenos como de su contexto socio-cultural (St. John et al. 2009). Como todo abordaje, los conceptos y métodos de la psicología social que se aplican en el ambiente rural tienen sus fortalezas y limitaciones. Por un lado, los modelos psicosociales superan las limitaciones del concepto del Homo economicus al reconocer la racionalidad limitada de los agentes frente a situaciones complejas y la influencia de factores emocionales en la formación de intenciones y la toma de decisiones (Kahneman 2003). Por otro lado, son factibles de ser integrados con modelos ecológicos y económicos, dado que reducen el comportamiento a un conjunto de categorías que, a priori, pueden ser analizadas de manera cuantitativa (Cooke et al. 2009). Sin embargo, estas últimas características confieren rigidez a los modelos psicosociales y limitan su capacidad para considerar aspectos del comportamiento no observables con los conceptos y métodos establecidos a priori.

El presente trabajo tiene como objetivo revisar teorías, conceptos, métodos de la psicología social aplicados en el ambiente rural del Chaco Seco para visibilizar y poner en debate sus aportes reales y potenciales a la investigación y gestión de ecosistemas y paisajes rurales sujetos a transformación por la agricultura. La hipótesis de trabajo es que las intervenciones dirigidas a modificar el comportamiento de los productores agropecuarios hacia decisiones más sustentables serán efectivas en la medida que la toma de decisiones de uso de la tierra sea analizada como un proceso complejo, dinámico y singular. Por lo tanto, se propone que con el aporte de la psicología social es posible diseñar intervenciones "a medida" de los distintos tipos de productores agropecuarios, cuya efectividad potencial es mayor que la de intervenciones que engloban a los productores bajo un mismo modelo de comportamiento. Esta revisión cuenta con tres secciones, cada una orientada a abordar aspectos distintos pero vinculados del comportamiento de los productores agropecuarios. Las secciones comienzan presentando conceptos y métodos en forma general y luego ilustran la aplicación de los mismos en estudios realizados en el Chaco Seco argentino. La primera sección se refiere a cómo explicar y predecir la formación de intenciones. La segunda se refiere a cómo describir y caracterizar la diversidad de identidades y su rol sobre los valores y las actitudes. La tercera se refiere a cómo evaluar y vincular los compromisos ecológicos y los conflictos de preferencias entre distintos tipos de agentes. Finalmente, se discute brevemente cómo integrar los aportes de la psicología social a la investigación y gestión inter- y trans-disciplinaria de los ecosistemas.

\section{REVISIÓN}

\section{El querer, deber y poder en la formación de intenciones}

La psicología social ha generado distintos marcos teóricos y analíticos para estudiar el proceso de toma de decisiones del individuo en su contexto social. Uno de ellos es la teoría del comportamiento planificado (TCP) propuesta por Ajzen (1991). La TCP propone un modelo conceptual para explicar la formación de intenciones, que es el proceso clave antes de tomar decisiones. Este modelo es apropiado para analizar comportamientos voluntarios, es decir, aquellos cuya ocurrencia está muy determinada por factores motivacionales endógenos del agente y comportamientos con alto costo, es decir, aquellos en los que se ponen en juego una significativa cantidad de tiempo, dinero, etc. (Steg and Vlek 2009). En su versión simplificada, la TCP propone que la intención es el precursor inmediato de la decisión, y está influenciada en forma directa por tres factores o constructos psico-sociales: la actitud, la norma subjetiva y el control del comportamiento (Figura 1). A su vez, el control del comportamiento afecta tanto la formación de la intención como la toma de la decisión. La evaluación del modelo mediante análisis cuantitativos de datos recolectados en encuestas estructuradas permite evaluar la influencia relativa de estos tres factores sobre la intención de realizar un comportamiento determinado (focal) que es voluntario y tiene un objetivo, acción asociada, contexto y temporalidad bien definida (principio de compatibilidad) (Ajzen 2011). Las revisiones de las evaluaciones empíricas de la TCP muestran que este modelo conceptual ofrece una explicación parsimoniosa de la formación de intenciones en contextos de toma de 


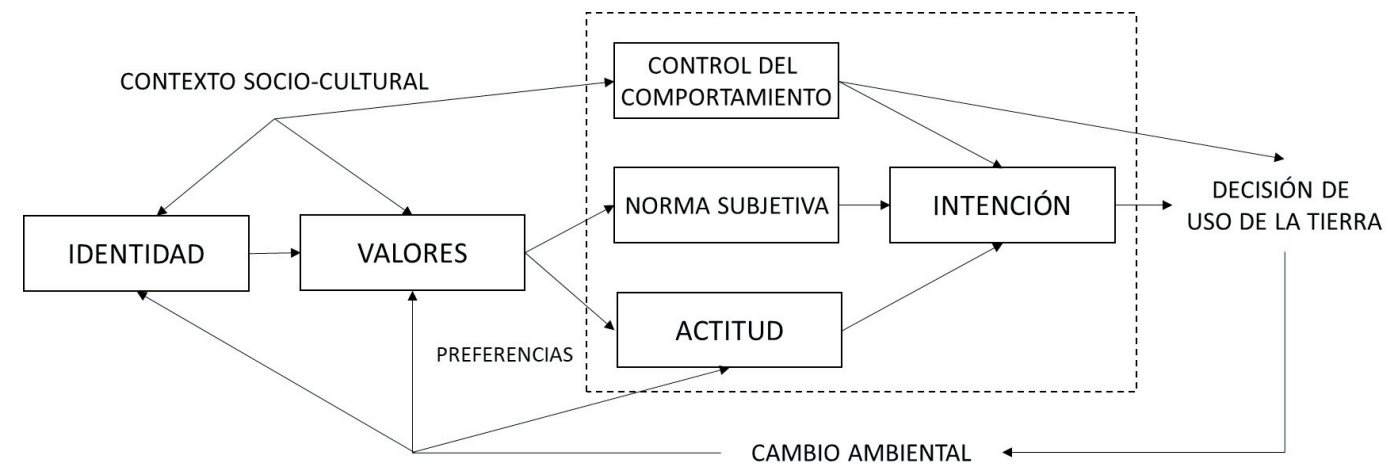

Figura 1. Modelo conceptual de los factores del comportamiento humano (constructos de la psicología social) que influyen sobre las decisiones de uso de la tierra y la influencia retroactiva que el cambio ambiental ejerce sobre ellos. Recuadro de línea punteada: modelo de la teoría del comportamiento planificado.

Figure 1. Conceptual model of human behavioral factors (constructs from social psychology) that influence land use decisions and the retroactive influence that environmental change exerts on them. Dotted line box: Theory of Planned Behavior model.

decisiones muy diversos, como el trabajo, la salud y el manejo de los recursos naturales (Armitage and Conner 2001).

En la TCP, la actitud consiste en la tendencia de un agente a valorar el resultado del comportamiento focal en forma favorable o desfavorable. Una actitud favorable hacia el comportamiento focal estará asociada a una fuerte intención de realizar dicho comportamiento, por lo que puede decirse que la actitud captura lo que el agente quiere o le interesa hacer. Por su parte, la norma subjetiva consiste en la percepción que el agente tiene sobre la opinión que sus pares y referentes tienen del comportamiento focal. Una percepción de que sus pares tienen una opinión positiva sobre el comportamiento focal estará asociada a una fuerte intención de realizar dicho comportamiento, por lo que puede decirse que la norma subjetiva captura lo que el agente cree que debe hacer. De esta manera, la influencia relativa de la actitud y la norma subjetiva sobre la intención permite estimar en qué medida el comportamiento es motivado por el interés propio o el interés colectivo del grupo de pertenencia del agente, respectivamente. Finalmente, el control del comportamiento consiste en la sensación que el sujeto tiene de ser capaz de ejecutar el comportamiento focal. Un fuerte control del comportamiento estará asociado a una fuerte intención de realizar dicho comportamiento y aumentará la probabilidad de que esa intención se materialice en una acción (Figura 1), por lo que puede decirse que el control del comportamiento captura lo que el agente cree que puede hacer.
Para poner a prueba la TCP es necesario instrumentar y hacer operativo el modelo conceptual descripto. El instrumento más comúnmente utilizado para evaluar la TCP es la encuesta estructurada, que consiste en un conjunto de ítems, cada uno de ellos compuesto por un enunciado afirmativo y una escala de respuesta tipo Likert. De esta manera, los distintos constructos psico-sociales de la TCP se miden mediante lo que los agentes declaran y no por medio de la observación de su comportamiento, lo cual representa una fuerte limitación a tener en cuenta (Cooke et al. 2009). Para la construcción de los ítems de la encuesta estructurada es recomendable realizar con anterioridad entrevistas con los sujetos de interés a fin de identificar los aspectos más salientes de cada constructo psico-social en relación al comportamiento focal. Por ejemplo, si el comportamiento focal es incorporar cultivos de cobertura en la rotación agrícola, los aspectos salientes que influyen sobre la actitud serán los beneficios y perjuicios asociados a esa acción (e.g., la regulación de malezas o la disminución de humedad en el suelo), los que influyen sobre la norma subjetiva serán los pares o referentes (e.g., productores vecinos o los técnicos del INTA) y los que influyen sobre el control del comportamiento serán una serie de barreras o facilitadores (e.g., disponibilidad de semillas, personal o maquinaria). Este tipo de información cualitativa que se obtiene en las entrevistas se usa luego para diseñar los enunciados afirmativos orientados a medir cada constructo psico-social. Siguiendo con el ejemplo, uno de los enunciados orientados a medir la actitud hacia la incorporación de 
cultivos de cobertura puede ser "Para mí, es importante incorporar cultivos de cobertura para regular las malezas", el cual será valorado por el productor agropecuario en una escala de acuerdo/desacuerdo conforme a la medida en que refleja su situación.

Mastrangelo et al. (2014) utilizaron la TCP con el objetivo de mejorar la comprensión de los factores motivacionales endógenos que influyen sobre las intenciones de los productores agropecuarios de conservar fragmentos remanentes de bosques nativos en sus predios en el oeste de la provincia de Chaco. Este estudio se realizó sobre una muestra de 89 productores agropecuarios con predios localizados en los departamentos de Almirante Brown y General Güemes, quienes fueron seleccionados y contactados con la colaboración de agentes de extensión rural del INTA y promotores del programa Cambio Rural. La muestra seleccionada comprendió productores con tamaño de predio entre 180 y 1764 ha, cubiertos en promedio por un $60 \%$ de bosque con ganadería extensiva, 15\% por cultivos anuales y $25 \%$ por pasturas perennes. Más del $90 \%$ dela muestra estuvo comprendida por familias o empresas familiares de productores nacidos y residentes en la región Chaqueña. Un $77.5 \%$ de la muestra reportó intenciones positivas hacia la conservación de los remanentes de bosque nativo en sus predios, mientras que el $22.5 \%$ restante reportó intenciones negativas. Los productores fueron visitados en dos oportunidades; en la primera se realizaron entrevistas semi-estructuradas y en la segunda se administró una encuesta estructurada. Ésta última fue construida en base a la información cualitativa obtenida en las entrevistas previas, y consistió de 27 ítems diseñados para cuantificar el valor de nueve constructos psico-sociales. El comportamiento focal fue construido siguiendo el principio de compatibilidad, en el que la intención fue consistentemente relevada en relación a la conservación (acción) de fragmentos remanentes de bosque nativo (objetivo) en el predio del productor encuestado (contexto) por el año venidero (la temporalidad). Uno de los objetivos específicos del estudio fue comparar el grado de ajuste y parsimonia entre tres modelos alternativos: a) el propuesto por la TCP , b) la TCP combinada con la teoría de la activación de la norma (Schwartz 1977), que incluía tres constructos adicionales (la norma personal, la conciencia del problema y la conciencia de las consecuencias), y c) la TCP combinada con la Teoría de la Activación de la Norma más los constructos de identidad y comportamiento pasado, los cuales fueron propuestos por Burton and Wilson (2006) como importantes factores influyentes del comportamiento de los productores agropecuarios.

El análisis cuantitativo de las respuestas de los productores agropecuarios se realizó utilizando modelos deecuaciones estructurales en dos pasos. Primero, se evaluó la validez de los nueve constructos psico-sociales mediante análisis factorial confirmatorio, el cual permite comprobar si las variables manifiestas (las respuestas a los enunciados) contribuyen a la variable latente (los constructos psico-sociales) tal como se propone en el modelo conceptual. Segundo, se evaluaron las relaciones propuestas entre los constructos psico-sociales en los tres modelos alternativos mediante análisis de trayectorias. Los resultados mostraron que el modelo simple propuesto por la TCP fue el que combinó mayor ajuste y parsimonia, confirmando el valor sinóptico de la TCP. El modelo simple de la TCP explicó virtualmente la misma cantidad de varianza que el modelo más complejo (41\% y 42\%, respectivamente), a la vez que su valor del criterio de Akaike fue $116 \%$ menor comparado con los otros dos modelos. Asimismo, los modelos resultantes mostraron que la norma subjetiva y la actitud fueron factores que consistentemente influyeron en forma positiva y significativa sobre la intención de los productores agropecuarios (coeficientes de regresión promedio igual a 0.33 y 0.31 , respectivamente). Por su parte, el control del comportamiento no influyó significativamente sobre las intenciones de conservar el monte nativo en sus predios. En cuanto a los factores integrados a la TCP, la identidad tuvo una fuerte influencia subyacente sobre la actitud y la norma subjetiva, y a través de éstos sobre la intención de conservar los fragmentos remanentes de bosque nativo.

Los resultados de este trabajo sugieren que los productores agropecuarios del Oeste de Chaco deciden si conservar o no en sus predios principalmente de acuerdo a lo que a ellos individualmente les interesa hacer con el bosque nativo en relación a los beneficios que perciben, pero sobre todo en respuesta a la opinión de sus pares, fundamentalmente otros productores, acerca de lo que ellos deben hacer con el bosque nativo. Por su parte, que el control del comportamiento no haya influido significativamente sobre sus intenciones de 
conservar el monte nativo sugiere que este tipo de productores en general cree que puede conservar, es decir, no percibe la presencia de barreras para ejecutar el comportamiento. Finalmente, la importancia de la identidad como factor subyacente a las actitudes, normas e intenciones sugiere que las expectativas de los productores agropecuarios acerca de cómo deberían comportarse como parte de su grupo de identidad, sea cual fuere éste, juegan un rol fundamental en las decisiones de conservar. Estos resultados pueden ser útiles para informar eldiseñodeintervenciones orientadas a modificar las intenciones negativas hacia la conservación del bosque nativo que persisten en una porción significativa de productores agropecuarios. Demostrar los beneficios de conservar los remanentes de bosque en sus predios permitiría aumentar la actitud positiva de los productores agropecuarios hacia su conservación. Establecer o reinstaurar normas subjetivas en los grupos de identidad según las que los productores agropecuarios que conserven remanentes del bosque nativo en sus predios tengan mejor reputación y mayor reconocimiento por sus pares puede representar una forma efectiva de revertir dichas intenciones.

\section{El rol de las identidades en los valores y actitudes}

Las personas tienden a valorar los ecosistemas y formar una actitud hacia la conservación en forma muy diferente. En el caso de los productores agropecuarios, éstos valores y actitudes suelen ser contrastantes y entrar en conflicto. La psicología social ofrece elementos para estudiar la formación de las actitudes, las cuales contribuyen fuertemente a configurar las intenciones, como se mostró en la sección anterior. Las actitudes suelen abordarse conceptualmente como la expresión (específica a un determinado comportamiento) de los valores, los cuales son descriptos con un carácter menos lábil y más duradero que las actitudes (Schultz and Zelezny 1999). Existen diversos esfuerzos tendientes a desarrollar abordajes amplios y pluralísticos para la valoración de los ecosistemas como insumo clave en la investigación y gestión de los mismos. Uno de ellos es llevado adelante en el marco de la Plataforma Intergubernamental sobre Biodiversidad y Servicios Ecosistémicos (IPBES), donde el concepto de "valor" puede ser referido tanto a un principio asociado a un determinado grupo cultural, una preferencia que alguien tiene sobre un determinado estado de un sistema, la importancia que se le asigna a un atributo de un objeto, proceso o comportamiento, o simplemente una medida (Pascual et al. 2017). En cualquier caso, los valores se refieren a conceptos abstractos y generales (ej. "la diversidad") sobre los que se desarrollan creencias (e.g., "el monte es diverso") en las que se sustenta la formación de actitudes (Ej. "me interesa conservar el monte"). En IPBES, los valores han sido clasificados en tres tipos: a) valor intrínseco (e.g., la diversidad por su propia existencia, independientemente del juicio del ser humano), b) valor instrumental (e.g., la diversidad como un medio para satisfacer necesidades o deseos del ser humano), y c) relacional (e.g., la diversidad porque permite el vínculo entre los seres humanos y de éstos con otros seres) (Chan et al. 2016).

La psicología social ha propuesto y demostrado cómo la interacción social y la internalización de normas en grupos de identidad juegan un rol fundamental en la formación de actitudes y valores (Figura 1). Dichas influencias surgen debido a que los sujetos tienden a compartir el significado simbólico de objetos y comportamientos, lo cual les confiere pertenencia a grupos y al desarrollo de una identidad (Stryker and Serpe 1994). Así, cada identidad está asociada a un conjunto de valores y actitudes compartidos por un grupo, la cual se mantiene mediante el propio reconocimiento de pertenencia de cada miembro (e.g., cuando declaran "yo soy un productor") y por la expectativa compartida entre los miembros de que cada uno de ellos debe comportarse de tal o cual manera (e.g., cuando declaran "los productores sólo producimos alimentos"). Sin embargo, la psicología social ha descripto cómo la identidad es múltiple y jerárquica, de manera que en cada actor social coexisten diversas identidades (e.g., ser productivista y ser conservacionista) las cuales se manifiestan con mayor o menor saliencia de acuerdo a su jerarquía y la situación contextual (e.g., ser productivista en las reuniones con grandes productores, pero conservacionista en las reuniones con extensionistas rurales) (Burton 2004). Como se mostró en la sección anterior, la incorporación del constructo "identidad" a la TCP aumenta la capacidad del modelo de explicar las actitudes, normas e intenciones. Por este motivo, otros estudios se han preguntado cuáles son los factores que determinan la alta influencia de la identidad en contextos de decisiones de conservación y manejo de ecosistemas, y han encontrado 
que el grado de conexión con la naturaleza (la sensación de ser parte de la naturaleza, Schultz 2001) y el apego al lugar (la sensación de pertenencia a un lugar, Raymond et al. 2010) influyen significativamente sobre la identidad (Lokhorst et al. 2014).

La diversidad de valores y actitudes de los productores agropecuarios, y la influencia de sus identidades sobre dicha diversidad, fueron evaluadas mediante entrevistas realizadas en 2013 a 36 productores agropecuarios con predios localizados en los alrededores de la localidad de Pampa del Infierno (departamento de Almirante Brown, provincia de Chaco) (Mastrangelo, datos no publicados). Los entrevistados fueron seleccionados de manera de capturar la heterogeneidad de productores agropecuarios presentes en el territorio, tanto en cuanto a tamaño de la producción como a la actividad productiva principal. La entrevista estuvo compuesta por dos partes. La primera tuvo un formato semi-estructurado y consistió de un conjunto de preguntas amplias y de respuesta abierta diseñadas para generar que el productor agropecuario describa, en sus propias palabras, sus prácticas y trayectorias de uso de la tierra, sus intenciones y actitudes hacia la conservación del bosque nativo, la percepción de sí mismo y su rol en ese contexto social, y la identificación de los beneficios que percibe provenientes de los ecosistemas. La segunda parte tuvo un formato estructurado y consistió de preguntas de respuesta cerrada diseñadas para cuantificar el valor otorgado a la provisión de 12 servicios ecosistémicos en dos sistemas de uso de la tierra (Figura 2). Los sistemas de uso de la tierra evaluados fueron un sistema de baja intensidad de uso que integra elementos del bosque nativo con pasturas y/o cultivos ("sistema integrado") y un sistema de alta intensidad de uso en el que el bosque nativo es reemplazado por pasturas y/o cultivos ("sistema intensivo"). Aquí se le pidió a los entrevistados que, utilizando una escala de -5 (extremadamente poco importante) a +5 (extremadamente importante), valoren la importancia cada uno de los 12 servicios ecosistémicos en cada uno de los sistemas.

Los datos obtenidos de la primera parte de la entrevista fueron utilizados para: a) clasificar y caracterizar a los entrevistados y construir una tipología de productores agropecuarios, b) definir un conjunto de beneficios que los entrevistados consideraban eran producidos por los ecosistemas y tenían influencia sobre su bienestar (servicios ecosistémicos). Las respuestas de los entrevistados fueron codificadas y se realizó una clasificación cualitativa sobre esos datos (Henry et al. 2015). La tipología de productores fue realizada clasificando a los entrevistados de acuerdo a sus atributos estructurales y sus prácticas y trayectorias de uso de la tierra declarados en la entrevista. La tipología de productores no fue construida en base a la percepción de sí mismo y su rol (identidad) o los valores, actitudes o intenciones declarados en las entrevistas por dos motivos. Primero, los factores motivacionales pueden ser diferentes entre los distintos sujetos, pero no necesariamente se excluyen unos a otros (Lokhorst et al. 2011). De hecho, en la próxima sección se muestra cómo valores y actitudes a priori incompatibles entre sí pueden coexistir y dar lugar a un continuum de preferencias. Segundo, la teoría de la auto-percepción (Bem 1972) propone que las personas construyen la imagen de sí mismo en base a sus decisiones y acciones, y no al revés. La clasificación de los 36 entrevistados dio lugar a seis clases de productores agropecuarios, los cuales fueron caracterizados sintéticamente en base al tamaño de su producción y su trayectoria de cambio de uso de la tierra (Figura 2): a) pequeños extensificadores $(4)$, b) pequeños mixtos (8), c) medianos diversificadores (7), d) medianos intensificadores (9), e) grandes expansionistas (3), y f) grandes intensificadores (5).

Los datos obtenidos de la segunda parte de la entrevista fueron utilizados para cuantificar y comparar el valor otorgado por parte de los productores agropecuarios a los 12 servicios ecosistémicos provistos por los sistema integrado e intensivo de uso de la tierra. La comparación de las medias de las valoraciones muestra dos patrones generales (Figura 2). Por un lado, la importancia otorgada a los servicios ecosistémicos provistos por el sistema integrado disminuye a medida que los productores agropecuarios tienen unidades de producción de mayor tamaño y hacen un uso más intensivo de la tierra (Figura 2a), mientras que la importancia relativa otorgada a los servicios ecosistémicos provistos por el sistema intensivo es similar entre los distintos tipos de productores agropecuarios (Figura 2b). Por otro lado, todos los tipos de productores agropecuarios (excepto los grandes expansionistas e intensificadores) tendieron a asignarle una importancia positiva y de similar magnitud a la gran mayoría de 


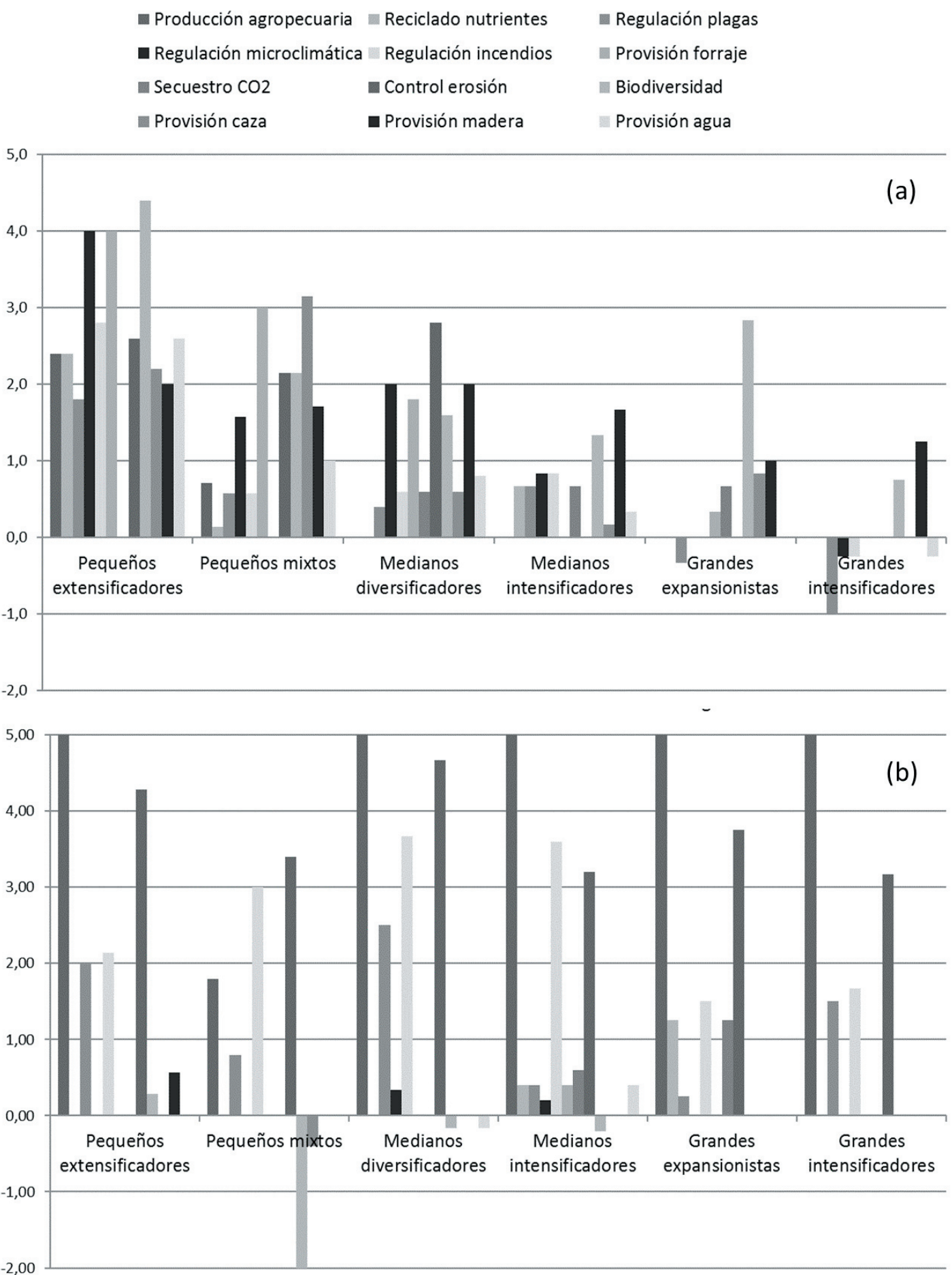

Figura 2. Valores asignados por seis tipos de productores agropecuarios del Chaco Seco argentino a un conjunto de doce servicios ecosistémicos provistos por un sistema de uso de la tierra integrado (a) e intensivo (b).

Figure 2. Values assigned by six types of farmers of the Argentine Dry Chaco to a set of twelve ecosystem services provided by an integrated (a) and an intensive (b) land use system. 
servicios ecosistémicos provistos por el sistema integrado, mientras que tendieron a asignar alta importancia a unos pocos servicios ecosistémicos y baja importancia a muchos servicios ecosistémicos provistos por el sistema intensivo.

Este estudio demuestra la utilidad de combinar conceptos y métodos de la sociología rural y la psicología ambiental. Por un lado, la construcción de tipologías de productores en base a sus estrategias productivas permite sintetizar y volver manejable la heterogeneidad social, económica y cultural presente en el territorio (Valbuena et al. 2008). Por otro lado, la valoración social de servicios ecosistémicos demuestra en forma cuantitativa, y sin necesidad de métricas económicas, la magnitud de las diferencias en los valores asignados a los servicios ecosistémicos por parte de los tipos de productores identificados, las cuales reflejan los intereses en disputa y el nivel de conflictividad real o potencial en el territorio. Esta es una primera aproximación a la valoración social de servicios ecosistémicos en el Chaco Seco que debe ser complementada con el estudio de los lenguajes (lógicas y categorías) de valoración propios de los actores territoriales, para evitar invisibilizar valores que no pueden capturarse con conceptos y métodos pautados por el investigador.

\section{Los compromisos ecológicos y conflictos de preferencias}

Distintos objetivos y resultados del uso de la tierra se ponen en juego y compiten en el proceso de toma de decisiones de los productores agropecuarios. Por esta razón, las actitudes involucradas en este proceso son múltiples e interactúan en forma particular de acuerdo al tipo de productor considerado. El estudio de las preferencias permite evaluar las relaciones de sinergia o conflicto entre actitudes hacia distintos objetivos del uso de la tierra. Identificar transiciones hacia sistemas de uso de la tierra capaces de balancear en forma más equitativa la producción de alimentos y la conservación de la biodiversidad es un objetivo clave de la gestión de los ecosistemas y paisajes. Para esto no alcanza con diagnosticar qué estados del sistema constituyen opciones de uso de la tierra que logran el mejor compromiso o mayor eficiencia en términos biofísicos, ya que la implementación de tales sistemas dependerá en última instancia de las decisiones de uso de la tierra de agentes individuales. Tales decisiones estarán influenciadas, como vimos, no sólo por la posibilidad material de realizarlo, sino que por sobre todo estarán determinadas por las actitudes y preferencias hacia la producción y conservación, las cuales muchas veces difieren entre productores agropecuarios con distintas identidades. De esta manera, resulta imperioso contar con un marco conceptual y analítico que permita identificar qué sistemas de uso de la tierra son a la vez biofísicamente posibles y socialmente deseables de realizar. Recientemente dicha herramienta ha sido propuesta conceptualmente por CavenderBares et al. (2015) y utilizada en el contexto del Chaco Seco argentino por Mastrangelo y Laterra (2015).

Dicho marco está basado en la integración de conceptos y métodos de la economía del comportamiento y la ecología de ecosistemas y paisajes. El primer paso del marco consiste en construir funciones de producción, una herramienta de la economía que se emplea para describir en qué medida es posible transformar recursos en bienes y servicios. Esta herramienta fue adoptada desde la ecología para describir cómo distintos grados de transformación de los ecosistemas producen diferentes niveles de provisión de servicios ecosistémicos (Paruelo et al. 2016). Las funciones de producción se utilizan en este marco para evaluar la relación de compromiso entre distintos servicios ecosistémicos a lo largo de un gradiente de transformación, lo cual da lugar a una curva de compromiso. Dicha curva muestra en qué medida una decisión de uso de la tierra que aumenta el nivel de provisión de un servicio ecosistémico genera un cambio en el nivel de provisión de otro, lo que permite identificar las opciones de uso de la tierra donde el compromiso entre dichos servicios ecosistémicos es menor $y$, por lo tanto, su producción es más eficiente; de allí que también se conozca como frontera de eficiencia o de posibilidad de producción (Smith et al. 2012). Una ventaja de evaluar compromisos mediante las funciones de producción consiste en que no es necesario valorar los servicios ecosistémicos usando métricas económicas, sino que cada uno se cuantifica en sus propias unidades (e.g., número de especies) (Polasky et al. 2008).

El segundo paso del marco consiste en construir curvas de indiferencia para los distintos tipos de productores agropecuarios $\mathrm{u}$ otro tipo de agentes involucrado en las decisiones de uso de la tierra. Las curvas de indiferencia son una herramienta de la economía del comportamiento utilizada para 
describir las preferencias de los consumidores respecto de bienes y servicios cuya producción está sujeta a una relación de compromiso; es decir, permite identificar la combinación más deseada de dichos bienes o servicios de entre todas las combinaciones que son biofísicamente posibles. Cuando se aplica a una relación de compromiso ecológica, las curvas de indiferencia muestran los estados del sistema (definidos por las combinaciones posibles de servicios ecosistémicos) en los que los niveles de satisfacción de los productores agropecuarios $\mathrm{u}$ otros grupos de identidad se mantienen invariables o indiferentes de acuerdo a sus preferencias. El tercer paso consiste en superponer gráficamente la curva de eficiencia y las curvas de indiferencia para identificar los puntos de intersección entre ambas curvas, los cuales indican los estados del sistema que constituyen opciones de uso de la tierra que son, a la vez, biofísicamente posibles y socialmente deseables para cada grupo de identidad. De esta manera, este marco conceptual y analítico incorpora el reconocimiento explícito de los conflictos de preferencia entre los grupos de interés en la búsqueda de soluciones de compromiso viables y sustentables de uso de la tierra (King et al. 2015).

Con el objetivo de identificar transiciones hacia sistemas sustentables de uso de la tierra que sean posibles desde lo biofísico y deseables desde el punto de vista social, Mastrangelo y Laterra (2015) relevaron las preferencias de 27 productores agropecuarios del este de Salta (departamento de San Martín) por combinaciones de niveles de biodiversidad (indicado por la actitud hacia retener los fragmentos de monte nativo en sus predios) y productividad agropecuaria (indicado por la actitud hacia remover dichos fragmentos). En el departamento de San Martín, la frontera agropecuaria se expande desde el pedemonte sub-húmedo hacia la llanura semiárida impulsada por productores agropecuarios de la región y extra-regionales que en los últimos 20 años comenzaron a utilizar tierras históricamente utilizadas por familias criollas y comunidades indígenas. Como en los casos anteriores, los entrevistados fueron seleccionados de manera de representar la heterogeneidad de productores agropecuarios presente en el territorio. Así, la muestra capturó un gradiente de intensidad de uso de la tierra que comprendió desde familias criollas que crían ganado vacuno en bosques de acceso abierto y animales de corral en sus "puestos", hasta grandes empresas agropecuarias con grandes superficies deforestadas y cultivadas crecientemente con pasturas implantadas para producir carne vacuna. Cada unidad de producción muestreada se visitó al menos en dos oportunidades durante 2010; una de ellas, para relevar las aves como indicador de biodiversidad, y otra para realizar una entrevista al productor. Esta entrevista estuvo compuesta de una parte semi-estructurada, orientada a caracterizar la unidad de producción, y otra estructurada, para relevar las preferencias del productor. Las opciones que se presentaron a los productores agropecuarios en la parte estructurada fueron combinaciones biofísicamente posibles de niveles de biodiversidad y productividad, obtenidos de la curva empírica de compromiso ecológico entre estos dos objetivos del uso de la tierra (Mastrangelo and Gavin 2012). Los productores encuestados fueron agrupados en base a sus preferencias declaradas y se encontraron tres grupos de productores bien definidos. El primer grupo ("multifuncionales de baja productividad") mostró preferencias por altos niveles de biodiversidad y niveles de productividad intermedios, y se caracterizó por un uso de la tierra diversificado y de baja intensidad, producciones familiares orientadas al autoconsumo y comercio de pequeña escala, en predios de dimensiones variables usualmente bajo formas de tenencia precaria o uso comunal. El segundo grupo ("multifuncionales de alta productividad") mostró preferencias por niveles intermedios de biodiversidad y niveles intermedios a altos de productividad, y se caracterizó por la integración de usos de la tierra (ganadería y agricultura) y de elementos nativos e introducidos (pasturas exóticas bajo monte nativo), producciones orientadas al comercio tanto regional como de exportación, en predios propios de tamaño mediano a grande manejados por sus dueños. El tercer grupo ("monofuncionales de alta productividad") mostró preferencias bajas a moderadas por la biodiversidad y consistentemente altas por la productividad, y se caracterizó por sistemas de uso de la tierra altamente simplificados y basados en el uso de insumos externos, producciones orientadas a la exportación de commodities agrícolas, en predios mayormente alquilados y manejados por personal contratado.

La Figura 2 muestra la curva de compromiso (curva cóncava con línea llena) y las curvas de indiferencia (curvas convexas) para los 
tres tipos de productores agropecuarios: multifuncionales de baja productividad (línea de guiones), multifuncionales de alta productividad (línea llena) y monofuncionales de alta productividad (línea de guiones y puntos). Los puntos bajo la curva de compromiso indican los estados de los sistemas de uso de la tierra reales definidos por la combinación de niveles de producción y conservación que fueron medidos en el terreno (Mastrangelo and Gavin 2012). Las letras indican los distintos puntos de intersección de las curvas de indiferencia con la curva de compromiso y permiten identificar barreras y oportunidades para la transición de los sistemas de uso de la tierra hacia la sustentabilidad. Promover la transición de los productores multifuncionales (tanto de baja como alta productividad) hacia los estados A y B sería deseable desde la perspectiva de la conservación. Sin embargo, la presión fuerte por incrementar los niveles de producción en el este de Salta, sumada a la vocación expansionista de los productores monofuncionales de alta productividad (representada por el estado E), aumentaría la vulnerabilidad de estos sistemas de baja productividad a ser desplazados y abandonar sus campos. Por lo tanto, al mirar el contexto socio-económico a escala regional, se observa que la probabilidad de que los sistemas multifuncionales aseguren su permanencia en el territorio y logren la sustentabilidad es mayor si logran realizar una transición hacia los estados C y D (flechas grises claro), en los que los niveles de producción serían significativamente mayores, sin que esto signifique una reducción significativa de los niveles de conservación. Por su parte, existe la posibilidad de que los productores monofuncionales de alta productividad transicionen hacia un sistema multifuncional sin resignar productividad (flecha gris oscura). Sin embargo, la intersección de las curvas para este grupo (punto E) indica que este tipo de productor, a diferencia de los otros dos grupos, no se encontraría satisfecho en estados de mayor multifuncionalidad, quizás en respuesta a la mayor complejidad asociada al manejo de sistemas más biodiversos.

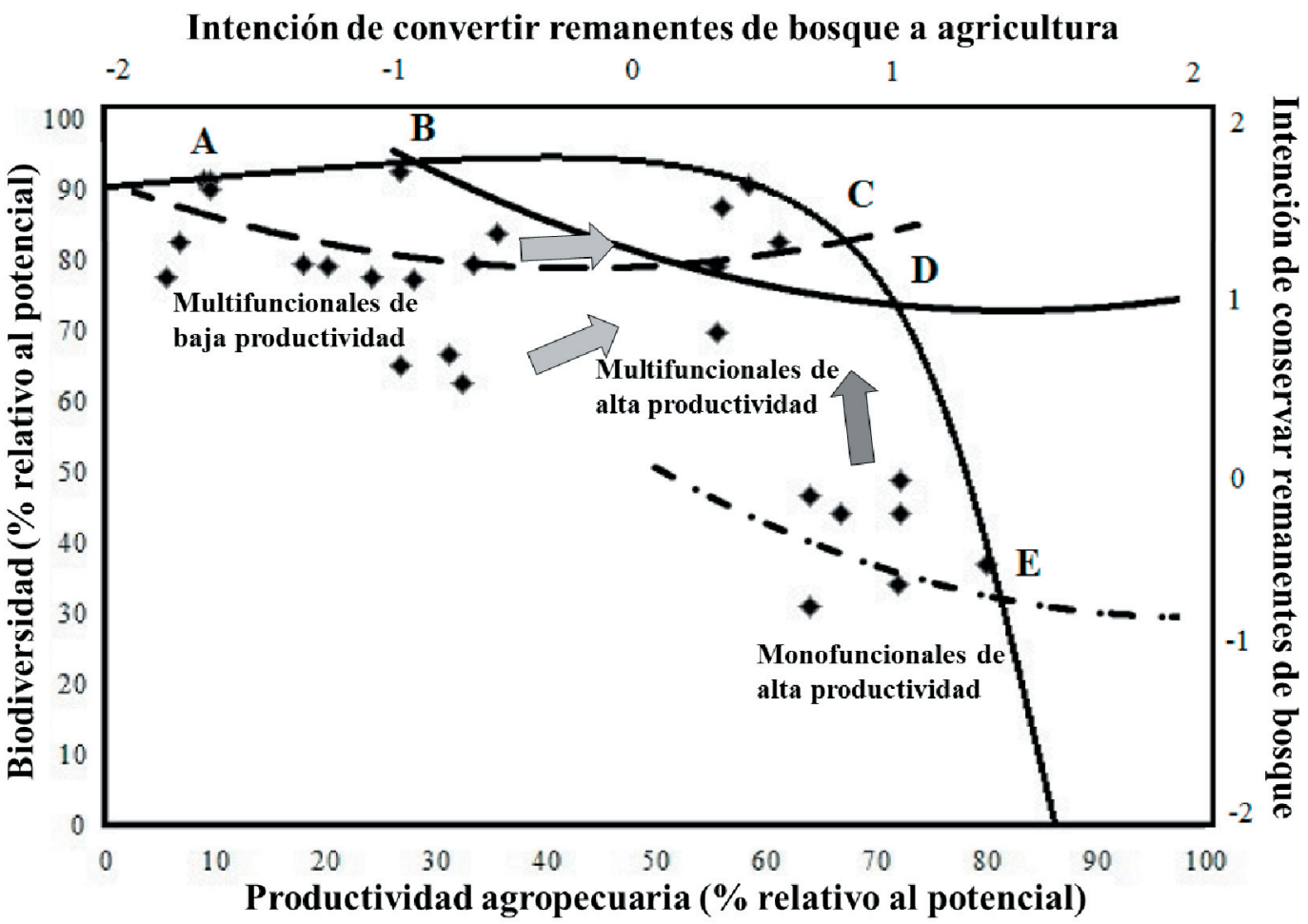

Figura 3. Intersección de las curvas de indiferencia para tres tipos distintos de productores agropecuarios y la curva empírica de compromiso entre conservación y producción (línea llena) en el Chaco Seco argentino. Las letras C y D indican estados del sistema que son, a la vez, biofísicamente posibles y socialmente deseables para los productores multifuncionales de baja productividad (línea de guiones), aunque posibles, pero no deseables, para los productores monofuncionales de alta productividad (línea de guiones y puntos).

Figure 3. Intersection of the indifference curves for three different types of farmers and the empirical trade-off curve between conservation and production (solid line) in the Argentine Dry Chaco. Letters C and D indicate system states that are both biophysically possible and socially desirable for multifunctional farmers of low productivity (dashed line), 


\section{DISCUSIÓN}

La presente revisión describe aportes que algunas aproximaciones desde las ciencias sociales pueden realizar en busca de mejorar nuestra capacidad de comprender e influir sobre las decisiones de uso de la tierra en paisajes rurales. Diversas ciencias sociales en general, y la psicología social en particular, ofrecen una gran diversidad de teorías, conceptos y métodos cuya aplicación de arroja luz sobre ciertos patrones y procesos sociales que subyacen a los cambios ambientales. Dado que el comportamiento humano es lo que, en última instancia, determina el estado de los ecosistemas, el conocimiento de dichos patrones y procesos sociales es clave para el diseño de intervenciones efectivas a fin de revertir la degradación ambiental. La teoría del comportamiento planificado demostró ser un modelo conceptual y analítico de gran valor sinóptico para comprender los factores motivacionales endógenos que influyen sobre la formación de las intenciones de conservar por parte de productores agropecuarios. Evaluar los factores que influyen sobre la formación de intenciones aporta información acerca de cuán (des)apropiado pueden ser los mecanismos de intervención. Por ejemplo, en caso de que un comportamiento esté motivado endógenamente es muy poco recomendable promoverlo mediante incentivos exógenos de tipo económico, ya que éste "reemplazo" puede erosionar las normas y actitudes que motivaban originalmente el comportamiento (de Snoo et al. 2013). Al demostrar la fuerte influencia de la norma subjetiva, la aplicación de la TCP permitió ir más allá de la noción de que los productores agropecuarios deciden si conservar o no únicamente en función de su interés personal y la maximización de sus beneficios, ya que las opiniones de sus pares y referentes jugaron un rol fundamental en su predisposición a conservar. Esto sugiere que establecer referentes con actitudes positivas a la conservación en los círculos de opinión de los productores agropecuarios puede ser una estrategia apropiada para modificar sus intenciones de conservar.

Por su parte, la valoración social de servicios ecosistémicos ofrece herramientas para revelar los distintos, y a veces contrapuestos, valores, actitudes y preferencias que los actores territoriales tienen respecto de los beneficios que derivan de los sistemas de uso de la tierra. Su aplicación permitió observar la diversidad de identidades y perfiles de valoración que coexisten en el territorio. Conocer esta heterogeneidad de pertenencias e intereses es importante para abordar procesos de negociación entre grupos con identidades y valores en conflicto, como aquellos que se producen en el marco del diseño e implementación de planes de ordenamiento territorial rural (Seghezzo et al. 2011). También permitió observar que sólo en algunos tipos de productores agropecuarios es posible suponer una correspondencia entre los valores asignados a los servicios ecosistémicos y sus trayectorias de uso de la tierra, indicando que los valores hacia los servicios ecosistémicos influyen en forma variable sobre las decisiones de uso de la tierra de acuerdo al tipo de productor agropecuario y el contexto. Por ejemplo, la alta valoración que los pequeños productores le otorgan a los servicios ecosistémicos que proveen los sistemas de alta intensidad de uso de la tierra (Figura 2) no tiene como correlato material una estrategia de intensificación, sino la de extensificar y diversificar la producción. Esto puede deberse a la presencia de barreras materiales que producen una desconexión entre los valores y las prácticas, o a que el abordaje utilizado no captura fielmente este tipo de valores en los productores agropecuarios.

Finalmente, la superposición de curvas de compromiso e indiferencia ofrece una herramienta simple y poderosa para vincular los compromisos ecológicos y los conflictos de preferencias e identificar barreras y oportunidades para que los productores agropecuarios hagan una transición hacia sistemas de uso de la tierra que sean a la vez eficientes y aceptables. Los resultados de su aplicación combinados con la evidencia generada en los anteriores estudios muestran una serie de implicancias para el diseño de intervenciones "a medida" del comportamiento de los productores agropecuarios, dirigidas a aumentar la sustentabilidad ambiental de su producción agropecuaria en el Chaco Seco argentino.

\section{Implicancias para el territorio}

El análisis conjunto de los estudios realizados en la región puede aportar elementos para evaluar oportunidades y barreras en la transición hacia sistemas sustentables de uso de la tierra, por ejemplo, mediante la comparación de los capitales (social, físico, institucional) que son necesarios para desarrollar los sistemas de uso de la tierra que los productores agropecuarios desean desarrollar (indicado por sus intenciones y preferencias) y los que efectivamente 
desarrollan (indicado por sus prácticas de manejo). En ecosistemas altamente frágiles comolos delChacoSeco argentino, la búsqueda de multifuncionalidad y el consecuente desarrollo de sistemas diversificados de uso de la tierra contribuyen de forma significativa a la sustentabilidad ambiental de los predios y los paisajes (Mastrangelo et al. 2014b). A su vez, dicha multifuncionalidad contribuye a la estabilidad de la producción y, por ende, a la sustentabilidad económica y social. En los estudios revisados, los productores multifuncionales de baja productividad mostraron una actitud positiva hacia transformar (en forma selectiva o no) pequeñas superficies de monte nativo para incrementar su productividad. Esta actitud coexistió con una consistente actitud positiva hacia mantener altos niveles de biodiversidad, lo cual se refleja en la alta intención de mantener la matriz de bosque en sus predios y el consecuente mantenimiento de la cantidad y calidad de hábitat. Esta preferencia representa una oportunidad para que este tipo de productores realice una transición hacia sistemas multifuncionales de alta productividad. Más aún, la norma subjetiva (lo que el sujeto cree que debería hacer) en este grupo de productores indica que dicha transición es socialmente deseable y aceptada. Sin embargo, a pesar de que la actitud y la norma subjetiva favorecen esta transición, los productores multifuncionales de baja productividad en general carecen de la capacidad o "control del comportamiento" para transformar incluso pequeñas superficies y utilizarlas para aumentar su productividad. Esta barrera está dada por las limitaciones de este tipo de productores en el acceso al capital institucional y físico necesario (títulos de propiedad, crédito, alambrados, maquinaria, etc.) para intensificar el uso de la tierra aún en pequeñas superficies.

Por el contrario, los productores monofuncionales de alta productividad no mostraron la actitud (no les interesa conservar o diversificar) ni la norma subjetiva (el grupo no comparte la expectativa de que los productores deberían conservar o diversificar) para realizar una transición hacia sistemas multifuncionales, aunque, en general, sí mostraron que tienen la capacidad de hacerlo si lo desean o si se sienten impulsados a hacerlo. De esta manera quedó demostrado que existen fuertes barreras psico-sociales para que este tipo de productores incorpore prácticas de uso de la tierra que le permitan aumentar sus niveles de biodiversidad, aun cuando cuenten con la certeza de que estas prácticas no ponen en riesgo su productividad. Se ha propuesto que los grandes productores difícilmente desarrollan una actitud positiva hacia la conservación debido a su lógica de maximización del beneficio económico, a su conexión débil con la naturaleza y a su desapego al lugar (Burton 2004). Sin embargo, también se vio que este tipo de productores es muy sensible a las opiniones de sus pares y de otros referentes, y es posible que modifiquen sus comportamientos en pos de mantener su imagen y reputación frente a su grupo de identidad y la sociedad (de Snoo et al. 2013). Por esta razón, la oportunidad para que este tipo de productores haga una transición hacia sistemas multifuncionales radica en la construcción de un capital social constituido por normas subjetivas por las que, a través de la presión de los pares referentes y de la opinión pública en general, se recompense con reconocimiento social (o "castigue" con sanción) a aquellos productores que adopten (o no) prácticas que promuevan la biodiversidad en los predios que manejan.

\section{Direcciones futuras}

Abordar la complejidad de los problemas ambientales requiere trabajar en forma intery transdisciplinaria, en especial cuando la investigación procura aportar a la gestión de los ecosistemas y paisajes. Esta revisión muestra algunos esfuerzos incipientes por integrar conceptos y métodos de las ciencias naturales y las ciencias sociales para abordar problemas reales como la deforestación y la pérdida de servicios ecosistémicos en fronteras agropecuarias del Chaco Seco argentino. Los estudios revisados sobre el comportamiento de los productores agropecuarios abrieron un conjunto amplio de nuevos interrogantes, algunos de los que se presentan aquí para estimular futuras investigaciones: ¿En qué medida el valor otorgado a los servicios ecosistémicos influye sobre las decisiones de uso de la tierra en los distintos tipos de productores agropecuarios? ¿Cómo influyen los cambios en el paisaje rural producidos por la expansión e intensificación agropecuaria sobre los valores, actitudes y preferencias hacia los ecosistemas y su conservación? ¿Cómo se retroalimentan los valores y las decisiones a través del cambio ambiental (Figura 1)? ¿Cuál es la influencia relativa de los factores motivacionales endógenos y los factores estructurales exógenos (contexto socio-cultural) sobre las decisiones de uso de la tierra? 
Agradecimientos. El autor agradece los valiosos aportes de un revisor y los comentarios de Alejandra Auer y Federico Weyland sobre una versión previa del manuscrico. Parte de este trabajo fue financiado con el proyecto
PICTO-2014-0046 “Bosques nativos, servicios ecosistémicos y bienestar humano en el Norte del Chaco Seco argentino: un abordaje interdisciplinar de la vulnerabilidad y los conflictos socioecológicos".

\section{REFERENCIAS}

Agrawal, A., and C. C. Gibson. 1999. Enchantment and disenchantment: The role of community in natural resource conservation. World Development 27:629-649.

Ajzen, I. 1991. The theory of planned behavior. Orgnizational Behavior and Human Decision Processes 50:179-211.

Ajzen, I. 2011. The theory of planned behavior: Reactions and reflections. Psychology and Health 26:1113-1127.

Armitage, C. J., and M. Conner. 2001. Efficacy of the Theory of Planned Behavior: a meta-analytic review. The British journal of social psychology / the British Psychological Society 40:471-499.

Bem, D. J. 1972. Self-Perception Theory. In L. Berkowitz (ed). Advances in Experimental Social Psychology 6:1-62.

Blaikie, P., and H. C. Brookfield (eds.). 1987. Land degradation and society. London: Methuen.

Brannstrom, C. 2009. South America's neoliberal agricultural frontiers: places of environmental sacrifice or conservation opportunity. AMBIO: A Journal of the Human Environment 38:141-149.

Brookfield, H. C. 1972. Intensification and disintensification in Pacific agriculture: A theoretical perspective. Pacific Viewpoint 13:30-48.

Boserup, E. 1965. The conditions of agricultural growth. Chicago: Aldine.

Burton, R. J. F. 2004. Reconceptualising the "behavioural approach" in agricultural studies: A socio-psychological perspective. Journal of Rural Studies 20:359-371.

Burton, R. J. F., and G. A. Wilson. 2006. Injecting social psychology theory into conceptualisations of agricultural agency: Towards a post-productivist farmer self-identity? Journal of Rural Studies 22:95-115.

Cáceres, D. M. 2015. Accumulation by dispossession and sociolenvironmental conflicts caused by the expansion of agribusiness in Argentina. Journal of Agrarian Change 15:116-147.

Cavender-Bares, J., S. Polasky, E. King, and P. Balvanera. 2015. A sustainability framework for assessing trade-offs in ecosystem services. Ecology and Society 20.

Chan, K. M. A., P. Balvanera, K. Benessaiah, M. Chapman, S. Díaz, E. Gómez-Baggethun, R. Gould, N. Hannahs, K. Jax, S. Klain, G. W. Luck, B. Martín-López, B. Muraca, B. Norton, K. Ott, U. Pascual, T. Satterfield, M. Tadaki, J. Taggart, and N. Turner. 2016. Opinion: Why protect nature? Rethinking values and the environment. Proceedings of the National Academy of Sciences 113:1462-1465.

Chayanov, A. V., D. Thorner, B. H. Kerblay, R. E. F. Smith, and American Economic Association. 1966. A.V. Chayanov on the theory of peasant economy. Homewood, Ill: Published for the American Economic Association by R.D. Irwin. American Economic Association translation series. Pp. 317.

Edwards-Jones, G. 2006. Modelling farmer decision-making: concepts, progress and challenges. Animal Science 82: 783-790.

Ellis, E. C., K. K. Goldewijk, S. Siebert, D. Lightman, and N. Ramankutty. 2010. Anthropogenic transformation of the biomes, 1700 to 2000. Global Ecology and Biogeography 19:589-606.

Grau, H. R., N. I. Gasparri, and T. M. Aide. 2008. Balancing food production and nature conservation in the Neotropical dry forests of northern Argentina. Global Change Biology 14:985-997.

Gurvich, D. E., D. Renison, and F. Barri. 2009. El rol del ecólogo ante la crisis ambiental actual. Ecología Austral 19: 233-238.

Hansen, M. C. C., P. V. Potapov, R. Moore, M. Hancher, S. A. a. Turubanova, A. Tyukavina, D. Thau, S. V. V. Stehman, S. J. J. Goetz, T. R. R. Loveland, a. Kommareddy, A. Egorov, L. Chini, C. O. O. Justice, J. R. G. R. G. Townshend, P. V. Patapov, R. Moore, M. Hancher, S. A. a. Turubanova, A. Tyukavina, D. Thau, S. V. V Stehman, S. J. J. Goetz, T. R. R. Loveland, A. Kommaredy, A. Egorov, L. Chini, C. O. O. Justice, and J. R. G. R. G. Townshend. 2013. High-Resolution Global Maps of. Science 342:850-854.

Hardin, G. 1968. Commons 13. Science 162:1243-1248.

Henry, D., A. B. Dymnicki, N. Mohatt, J. Allen, and J. G. Kelly. 2015. Clustering Methods with Qualitative Data: A Mixed-Methods Approach for Prevention Research with Small Samples. Prevention science: the official journal of the Society for Prevention Research 16:1007-1016.

Kahneman, D. 2003. A perspective on judgment and choice: Mapping bounded rationality. American Psychologist 58:697-720.

King, E., J. Cavender-Bares, P. Balvanera, T. H. Mwampamba, and S. Polasky. 2015. Trade-offs in ecosystem services and varying stakeholder preferences: Evaluating conflicts, obstacles, and opportunities. Ecology and Society 20.

Levine, J., K. M. A. Chan, and T. Satterfield. 2015. From rational actor to efficient complexity manager: Exorcising the ghost of Homo economicus with a unified synthesis of cognition research. Ecological Economics 114:22-32.

Lokhorst, A. M., C. Hoon, R. le Rutte, and G. de Snoo. 2014. There is an I in nature: The crucial role of the self in nature conservation. Land Use Policy 39:121-126. 
Lokhorst, A. M., H. Staats, J. Van Dijk, E. Van Dijk, and G. De Snoo. 2011. What's in it for Me? motivational differences between farmers' subsidised and non-subsidised conservation practices. Applied Psychology 60:337-353.

Meyfroidt, P. 2013. Environmental cognitions, land change, and social-ecological feedbacks: An overview. Journal of Land Use Science 8:341-367.

Mastrangelo, M. E., and M. C. Gavin. 2012. Trade-Offs between Cattle Production and Bird Conservation in an Agricultural Frontier of the Gran Chaco of Argentina. Conservation Biology 26(6):1040-1051. doi: 10.1111/j.15231739.2012.01904.x.

Mastrangelo, M. E., M. C. Gavin, P. Laterra, W. L. Linklater, and T. L. Milfont. 2014a. Psycho-Social Factors Influencing Forest Conservation Intentions on the Agricultural Frontier. Conservation Letters 7(2):103-110.

Mastrangelo, M. E., F. Weyland, S. H. Villarino, M. P. Barral, L. Nahuelhual, and P. Laterra. 2014b. Concepts and methods for landscape multifunctionality and a unifying framework based on ecosystem services. Landscape Ecology 29(2):345-358.

Mastrangelo, M. E., and P. Laterra. 2015a. From biophysical to social-ecological trade-offs: Integrating biodiversity conservation and agricultural production in the Argentine Dry Chaco. Ecology and Society 20(1):20.

Mastrangelo, M. E., F. Weyland, L. P. Herrera, S. H. Villarino, M. P. Barral, and A. D. Auer. 2015b. Ecosystem services research in contrasting socio-ecological contexts of Argentina: Critical assessment and future directions. Ecosystem Services 16:63-73.

Morello, J., W. Pengue, and A. Rodríguez. 2005. Etapas de uso de los recursos y desmantelamiento de la biota del Chaco. Fronteras 4:1-17.

Paruelo, J. M. 2016. El papel de la Ciencia en el proceso de Ordenamiento Territorial (y en otras cuestiones vinculadas con problemas ambientales). Ecología Austral 26:51-58.

Pascual, U., P. Balvanera, S. Díaz, G. Pataki, E. Roth, M. Stenseke, R. Watson, E. Dessane, S. Breslow, M. Islar, E. Kelemen, H. Keune, V. Maris, W. Pengue, M. Quaas, S. Subramanian, H. Wittmer, A. Mohamed, Y. Al-Hafedh, S. Asah, P. Berry, E. Bilgin, C. Bullock, D. Cáceres, C. Golden, E. Gómez-Baggethun, D. González-Jiménez, J. Houdet, R. Kumar, P. May, A. Mead, P. O'Farrell, D. Pacheco-Balanza, R. Pandit, R. Pichis-Madruga, F. Popa, S. Preston, H. Saarikoski, B. Strassburg, M. Verma, N. Yagi, S. Ahn, E. Amankwah, H. Daly-Hassen, E. Figueroa, K. Ma, M. van den Belt, and F. Wickson. 2017. Valuing nature's contributions to people: The IPBES approach. Current Opinion in Environmental Sustainability 26-27:7-16.

Polasky, S., E. Nelson, J. Camm, B. Csuti, P. Fackler, E. Lonsdorf, C. Montgomery, D. White, J. Arthur, B. Garber-Yonts, R. Haight, J. Kagan, A. Starfield, and C. Tobalske. 2008. Where to put things? Spatial land management to sustain biodiversity and economic returns. Biological Conservation 141:1505-1524.

Poppenborg, P., and T. Koellner. 2013. Do attitudes toward ecosystem services determine agricultural land use practices? An analysis of farmers' decision-making in a South Korean watershed. Land Use Policy 31:422-429.

Queenborough, E. H. A. Mattison, A. P. Bailey, D. L. Sandars, A. R. Graves, J. Morris, P. W. Atkinson, P. Trawick, R. P. Freckleton, A. R. Watkinson, and W. J. Sutherland. 2009. Integrating socio-economics and ecology: A taxonomy of quantitative methods and a review of their use in agro-ecology. Journal of Applied Ecology 46:269-277.

Raymond, C. M., G. Brown, and D. Weber. 2010. The measurement of place attachment: Personal, community, and environmental connections. Journal of Environmental Psychology 30:422-434.

Roy Chowdhury, R., and B. L. Turner. 2006. Reconciling agency and structure in empirical analysis: smallholder land use in the southern Yucatán, Mexico. Annals of the Association of American Geographers 96:302-322.

Scheffer, M., W. Brock, and F. Westley. 2000. Socioeconomic mechanisms preventing optimum use of ecosystem services: An interdisciplinary theoretical analysis. Ecosystems 3:451-471.

Schultz, P. 2001. The structure of environmental concern: Concern for self, other people, and the biosphere. Journal of Environmental Psychology 21:327-339.

Schultz, P., and L. Zelezny. 1999. Values as predictors of environmental attitudes: Evidence for consistency across 14 countries. Journal of Environmental Psychology 19:255-265.

Schwartz, S. H. 1977. Normative Influences on Altruism. Advances in Experimental Social Psychology 10:221-279.

Seghezzo, L., J. N. Volante, J. M. Paruelo, D. J. Somma, E. C. Buliubasich, H. E. Rodriguez, S. Gagnon, and M. Hufty. 2011. Native Forests and Agriculture in Salta (Argentina): Conflicting Visions of Development. The Journal of Environment and Development 20:251-277.

Smith, F. P., R. Gorddard, A. P. N. House, S. McIntyre, and S. M. Prober. 2012. Biodiversity and agriculture: Production frontiers as a framework for exploring trade-offs and evaluating policy. Environmental Science and Policy 23:8594.

de Snoo, G. R., I. Herzon, H. Staats, R. J. F. Burton, S. Schindler, J. van Dijk, A. M. Lokhorst, J. M. Bullock, M. Lobley, T. Wrbka, G. Schwarz, and C. J. M. Musters. 2013. Toward effective nature conservation on farmland: Making farmers matter. Conservation Letters 6:66-72.

Steg, L., and C. Vlek. 2009. Encouraging pro-environmental behavior: An integrative review and research agenda. Journal of Environmental Psychology 29:309-317.

St John, F. A., G. Edwards-Jones, and J. P. Jones. 2011. Conservation and human behavior: lessons from social psychology. Wildlife Research 37:658-667.

Stryker, S., and R. T. Serpe. 1994. Identity salience and psychological centrality: Equivalent, overlapping, or complementary concepts? Social Psychology Quarterly 57:16-35.

Valbuena, D., P. H. Verburg, and A. K. Bregt. 2008. A method to define a typology for agent-based analysis in regional 
land-use research. Agriculture, Ecosystems and Environment 128:27-36.

Vallejos, M., J. N. Volante, M. J. Mosciaro, L. M. Vale, M. L. Bustamante, and J. M. Paruelo. 2015. Transformation dynamics of the natural cover in the Dry Chaco ecoregion: A plot level geo-database from 1976 to 2012. Journal of Arid Environments 123:3-11.

Volante, J. N., Alcaraz-Segura, D., Mosciaro, M. J., Viglizzo, E. F., and Paruelo, J. M. 2012. Ecosystem functional changes associated with land clearing in NW Argentina. Agriculture, Ecosystems and Environment 154:12-22. 